\title{
Unintentional Dual Benefit: Improvement in Secondary Polycythemia in a Patient Receiving Chemotherapy for Metastatic Clear Cell Renal Cell Carcinoma
}

\author{
Brooke Kania, Erinie Mekheal, Sherif Roman, Nader Mekheal, Vinod Kumar, Michael Maroules \\ St. Joseph's University Medical Center, Paterson, NJ, USA
}

Received: $14 / 12 / 2021$

Accepted: $19 / 01 / 2022$

Published: $18 / 02 / 2022$ How to cite this article: Kania B, Mekheal E, Roman S, Mekheal N, Kumar V, Maroules M. Unintentional dual benefit: improvement in secondary
polycythemia in a patient receiving chemotherapy for metastatic clear cell renal cell carcinoma. EJCRIM 2022;9:doi:10.12890/2022_003125.

Conflicts of Interests: The Authors declare that there are no competing interests.

This article is licensed under a Commons Attribution Non-Commercial 4.0 License

\section{ABSTRACT}

Complete blood counts are frequently collected from cancer patients, but laboratory findings may be misleading. Secondary polycythemia can occur in renal cell carcinoma (RCC) due to erythropoietin (EPO) stimulation. Therefore, complete blood counts should be closely monitored to prevent complications such as thrombosis. We discuss the case of a 47-year-old man with metastatic RCC who presented with secondary polycythemia that improved with chemotherapy. His secondary erythrocytosis was anticipated, but his haemoglobin levels were lower than expected after therapy. This article discusses the treatment and diagnosis of secondary polycythemia in patients with RCC.

\section{KEYWORDS}

Metastatic renal cell carcinoma, everolimus, paraneoplastic syndrome, secondary polycythemia, EPO-producing tumour

\section{LEARNING POINTS}

- Haemoglobin and haematocrit levels should be closely monitored in renal cell carcinoma (RCC) patients as they may develop secondary polycythemia as a result of their malignancy.

- Secondary polycythemia can be managed with chemotherapy and immunotherapy in RCC, with anti-cancer agents preventing the need for phlebotomy.

- Chemotherapy may benefit RCC patients by decreasing tumour burden, preventing progression, and by lowering haemoglobin levels, thus improving secondary polycythemia.

\section{CASE DESCRIPTION}

The patient was a 47-year-old man with a history of clear cell RCC after left-sided nephrectomy (Fig.1). He was initially staged T2aNxM0, but 3 years after surgery, cancer recurred in the pancreatic tail and in lung nodules. Two years after this, CT of the chest, abdomen and pelvis was significant for disease progression, and the patient was initiated on pembrolizumab and axitinib. He received 13 cycles of pembrolizumab and daily axitinib, which resulted in significant improvement in his metastatic pulmonary lesions and secondary polycythemia.

However, he developed dysgeusia, nausea, non-bloody diarrhoea and asthenia, suspected secondary to axitinib, which was discontinued. He completed two additional cycles of pembrolizumab, completing a total of 15 cycles of immunotherapy. During the patient's final cycle of pembrolizumab, he was hospitalized for suspected pneumonitis due to immunotherapy and pembrolizumab was discontinued. 


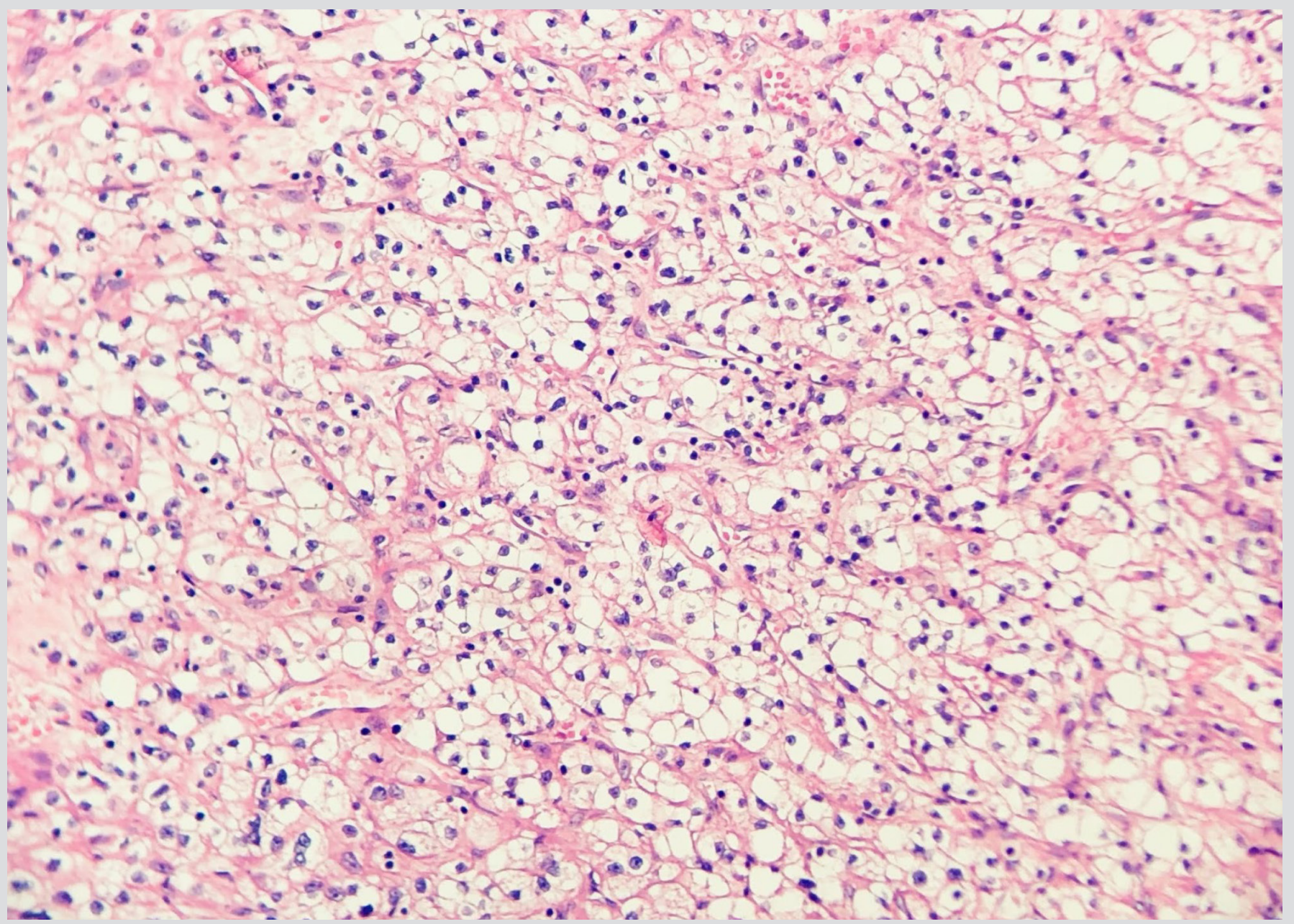

Figure 1. Renal cell carcinoma (clear cell), optically clear cells with round, uniform nuclei (due to glycogen content), arranged in nests in 'chicken wire' vasculature, Grade 1.

Sometimes, delicate capillaries rupture during surgical manipulation, resulting in the formation of 'blood lakes' within tumour cell nests. H\&E stain

\begin{tabular}{|c|c|c|}
\hline Cycle & Treatment & Hgb (g/d)/Hct (\%) \\
\hline Cycle 1 & Axitinib and pembrolizumab & $17.0 / 49.0$ \\
\hline Cycle 1 & Axitinib and pembrolizumab & $17.0 / 49.0$ \\
\hline Cycle 2 & Axitinib and pembrolizumab & $16.8 / 50.2$ \\
\hline Cycle 3 & Axitinib and pembrolizumab & $17.4 / 51.3$ \\
\hline Cycle 4 & Axitinib and pembrolizumab & $17.1 / 53.1$ \\
\hline Cycle 5 & Axitinib and pembrolizumab & $17.0 / 51.3$ \\
\hline Cycle 6 & Axitinib and pembrolizumab & $16.8 / 54.2$ \\
\hline Cycle 7 & Axitinib and pembrolizumab & $17.1 / 51.62$ \\
\hline Cycle 8 & Axitinib and pembrolizumab & $17.2 / 50.8$ \\
\hline
\end{tabular}

\begin{tabular}{|c|c|c|}
\hline Cycle & Treatment & Hgb (g/dl)/Hct (\%) \\
\hline Cycle 9 & Axitinib and pembrolizumab & $17.8 / 54.1$ \\
\hline Cycle 10 & Axitinib and pembrolizumab & $18.0 / 59.6$ \\
\hline Cycle 11 & Axitinib and pembrolizumab & $16.8 / 48.5$ \\
\hline Cycle 12 & Axitinib and pembrolizumab & $16.0 / 48.6$ \\
\hline Cycle 13 & Axitinib and pembrolizumab & $15.3 / 48.2$ \\
\hline & Axitinib only & $15.3 / 48.2$ \\
\hline Cycle 14 & Pembrolizumab only & $14.4 / 43.0$ \\
\hline Cycle 15 & Pembrolizumab only & $14.0 / 41.8$ \\
\hline Cycle 8 & Axitinib and pembrolizumab & $17.2 / 50.8$ \\
\hline
\end{tabular}

Table 1. Haemoglobin ( $\mathrm{Hgb}$ ) and haematocrit (Hct) levels during treatment with pembrolizumab (cycles 1-15) and daily axitinib (cycles 1-13). Normal Hgb: 12.0-16.0 g/dl; normal Hct: $36.0-46.0 \%$. 


\begin{tabular}{|c|c|}
\hline \multicolumn{2}{|c|}{ Treatment with daily everolimus } \\
\hline & Hgb (g/dl)/Hct (\%) \\
\hline First month & $14.1 / 42.6$ \\
\hline Second month & $14.9 / 45.2$ \\
\hline Third month & $14.7 / 43.0$ \\
\hline Fourth month & $12.4 / 36.4$ \\
\hline
\end{tabular}

Table 2. Haemoglobin (Hgb) and haematocrit (Hct) levels during treatment with everolimus every day for 4 months. Normal $\mathrm{Hgb}: 12.0-16.0 \mathrm{~g} / \mathrm{dl}$; normal Hct: 36.0-46.0 g/dl.

At the beginning of his treatment with pembrolizumab and axitinib, the patient's haemoglobin levels and haematocrit were consistently elevated, suspected to be secondary to carcinoma-related excess EPO production (Table 1). These haemoglobin levels met $2016 \mathrm{WHO}$ criteria for polycythemia as values were above the threshold of $16.5 \mathrm{~g} / \mathrm{dl}$. The EPO level was also found to be higher than the upper limit of normal, with a value of $19.6 \mathrm{mU} / \mathrm{ml}$ (normal 2.6-18.5). The patient denied bleeding, the faecal occult blood test was negative, and colonoscopy was negative for gastrointestinal bleeding. Laboratory results did not show findings consistent with iron deficiency anaemia.

Due to disease progression, the patient was initiated on everolimus. His haemoglobin and haematocrit continue to trend downwards to within normal limits (Table 2).

\section{DISCUSSION}

Increased EPO levels are seen in some patients with RCC. Elevated EPO as a paraneoplastic process has been demonstrated in approximately one-third of RCC patients in two separate studies ${ }^{[1,2]}$. In another study, approximately $66 \%$ of RCC patients had elevated EPO levels; however, only $8 \%$ had polycythemia due to EPO overproduction ${ }^{[3]}$. The prevalence is high enough that EPO has been studied as a tumour marker for RCC, with decreased EPO levels after nephrectomy and increased EPO levels with tumour recurrence in patients who had EPO elevation at the time of diagnosis of the primary tumour ${ }^{[3,4]}$. RCC patients with polycythemia have shown partial or complete response to anti-cancer therapy and may have a higher response to some therapies such as IL-2 and alpha-interferon treatment than those without polycythemia ${ }^{[5]}$. In one study evaluating 37 patients, those with tumour histological staining positive for EPO were over twice as likely to die as those with negative staining, signifying a poor prognosis in this subset of patients ${ }^{[6]}$.

RCC can lead to a paraneoplastic syndrome called 'secondary polycythemia'. This phenomenon is caused by independent EPO production resulting from VHL-mutation-based modified regulation of hypoxia-inducible transcription factors ${ }^{[7]}$. Our patient's EPO levels were consistently above the upper limit of normal throughout the course of his cancer. In general, patients with primary polycythemia vera tend to have low EPO levels; however, elevated EPO levels are $98 \%$ specific for secondary erythrocytosis ${ }^{[8]}$. It is crucial to monitor secondary polycythemia given the consequences of uncontrolled polycythemia. In terms of incidence, it is estimated that haematologists see one new patient with polycythemia vera for approximately every 10 patients with relative or secondary polycythemia ${ }^{[9]}$. Our patient was found to have elevated haemoglobin and haematocrit levels approaching the criteria for polycythemia. However, after treatment was initiated, haemoglobin dropped to low to normal levels, indicating the therapeutic effect of the immunotherapy and chemotherapy.

Polycythemia is an important diagnosis as patients with primary polycythemia vera have an increased risk of arterial and venous thrombosis and of major haemorrhage ${ }^{[10]}$. Those with malignancy already have an increased risk of thrombosis ${ }^{[11]}$, and therefore erythrocytosis due to a tumour creates an even greater risk of thrombotic events. The therapeutic effect of combination immunotherapy and chemotherapy in our patient helped avoid multiple phlebotomies and ultimately prevented thrombotic, haemorrhagic and/or cardiovascular complications. In addition, our patient had initial hypertension, which improved following anti-cancer treatment, likely due to the improvement in his erythrocytosis.

In our case, the patient received chemotherapy and immunotherapy with axitinib (tyrosine kinase inhibitor) and pembrolizumab (inhibits binding of PD-1 ligands by binding to the PD-1 receptor on T-cells). However, pembrolizumab has the serious side effect of autoimmune haemolytic anaemia, which may be due to auto-antibody formation, T-cell clonal formation, and decreased T-cell regulation ${ }^{[12]}$. Our patient did not exhibit findings of haemolysis, but his haemoglobin levels decreased after treatment was initiated. Axitinib ultimately helped inhibit the patient's tumour progression while simultaneously controlling his polycythemia. 
After discontinuing pembrolizumab and axitinib, our patient received everolimus, an mTOR inhibitor. This agent can cause significant anaemia in patients, leading to morbidity and treatment interruption, and eventually requiring discontinuation of therapy for some patients ${ }^{[13]}$. Although our patient developed bone marrow suppression leading to anaemia caused by everolimus, this ultimately benefited him, as this further treated his polycythemia without the need for phlebotomy while decreasing his tumour burden.

As the patient received three different regimens for his cancer, our analysis is limited in identifying which specific regimen most impacted on his EPO overproduction-induced polycythemia. Studies of additional cases are necessary to confirm these results and determine whether EPO levels can be used as tumour markers in this patient population.

\section{REFERENCES}

1. Ljungberg B, Rasmuson T, Grankvist K. Erythropoietin in renal cell carcinoma: evaluation of its usefulness as a tumor marker. Eur Urol 1992;21:160-3.

2. Michael A, Politi E, Havranek E, Corbishley C, Karapanagiotou L, Anderson C, et al. Prognostic significance of erythropoietin expression in human renal cell carcinoma. BJU Int 2007;100(2):291-4.

3. Palapattu GS, Kristo B, Rajfer J. Paraneoplastic syndromes in urologic malignancy: the many faces of renal cell carcinoma. Rev Urol 2002;4(4):163.

4. Murphy GP, Kenny GM, Mirand EA. Erythropoietin levels in patients with renal tumors or cysts. Cancer 1970;26(1):191-4.

5. Janik JE, Sharfman WH, Smith JW, Longo DL, Sznol M, Urba WJ, et al. Erythropoietin production. A potential marker for interleukin-2/interferon-responsive tumors. Cancer 1993;72(9):2656-9.

6. Michael A, Politi E, Havranek E, Corbishley C, Karapanagiotou L, Anderson C. Prognostic significance of erythropoietin expression in human renal cell carcinoma. BJU Int 2007;100(2):291-4.

7. Bunn HF, Gu J, Huang LE, Park JW, Zhu H. Erythropoietin: a model system for studying oxygen-dependent gene regulation. J Exp Biol 1998;201(8):1197-1201.

8. Messinezy M, Westwood NB, El-Hemaidi I, Marsden JT, Sherwood RS, Pearson TC. Serum erythropoietin values in erythrocytoses and in primary thrombocythaemia. Br J Haematol 2002;117(1):47-53.

9. Berlin NI. Diagnosis and classification of the polycythemias. Semin Hematol 1975 Oct;12(4):339-51.

10. Tefferi A, Rumi E, Finazzi G, Gisslinger H, Vannucchi AM, Rodeghiero F, et al. Survival and prognosis among 1545 patients with contemporary polycythemia vera: an international study. Leukemia 2013;27(9):1874-81.

11. Donati MB, Falanga A. Pathogenetic mechanisms of thrombosis in malignancy. Acta Haematol 2001;106(1-2):18-24.

12. Tanios GE, Doley PB, Munker R. Autoimmune hemolytic anemia associated with the use of immune checkpoint inhibitors for cancer: 68 cases from the Food and Drug Administration database and review. Eur J Haematol 2019;102(2):157-62.

13. Shameem R, Hamid MS, Wu S. Risk of anemia attributable to everolimus in patients with cancer: a meta-analysis of randomized controlled trials. Anticancer Res 2015;35(4):233340. 